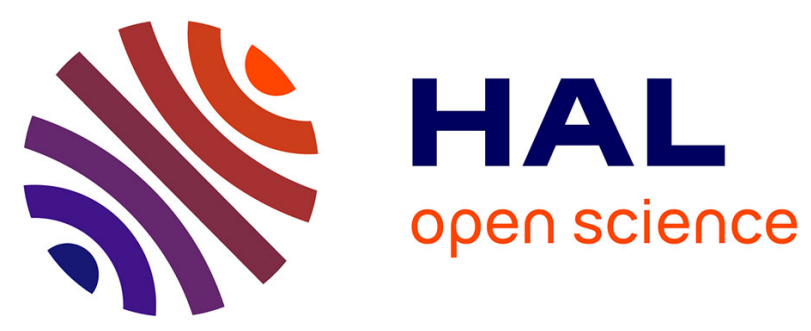

\title{
3D analysis of gelatin gel networks from transmission electron microscopy imaging
}

M. Djabourov, N. Bonnet, H. Kaplan, N. Favard, P. Favard, J. Lechaire, M. Maillard

\section{- To cite this version:}

M. Djabourov, N. Bonnet, H. Kaplan, N. Favard, P. Favard, et al.. 3D analysis of gelatin gel networks from transmission electron microscopy imaging. Journal de Physique II, 1993, 3 (5), pp.611-624. 10.1051/jp2:1993155 . jpa-00247858

HAL Id: jpa-00247858

https://hal.science/jpa-00247858

Submitted on 1 Jan 1993

HAL is a multi-disciplinary open access archive for the deposit and dissemination of scientific research documents, whether they are published or not. The documents may come from teaching and research institutions in France or abroad, or from public or private research centers.
L'archive ouverte pluridisciplinaire HAL, est destinée au dépôt et à la diffusion de documents scientifiques de niveau recherche, publiés ou non, émanant des établissements d'enseignement et de recherche français ou étrangers, des laboratoires publics ou privés. 
Classification

Physics Abstracts

$82.70 \mathrm{G}-61.16 \mathrm{D}$

\title{
3D analysis of gelatin gel networks from transmission electron microscopy imaging
}

\author{
M. Djabourov $\left({ }^{1, *}\right), \quad$ N. Bonnet $\left({ }^{2}\right)$, \\ H. Kaplan $\left({ }^{2}\right)$, \\ N. Favard $\left({ }^{3}\right)$, \\ P. Favard ${ }^{\dagger}\left({ }^{3}\right)$ \\ J. P. Lechaire $\left({ }^{3}\right)$ and M. Maillard $\left({ }^{3}\right)$ \\ (1) Ecole de Physique et Chimie, CNRS URA 857, 10 rue Vauquelin, 75231 Paris Cedex 05, \\ France \\ ( $\left.{ }^{2}\right)$ Unité INSERM 314, Université de Reims, 21 rue Clément Ader, 51100 Reims, France \\ (3) Centre de Biologie Cellulaire CNRS, 67 rue Maurice Günsbourg, 94205 Ivry-sur-Seine \\ Cedex, France
}

(Received 29 December 1992, accepted in final form 11 February 1993)

\begin{abstract}
This paper presents a quantitative analysis of the structural parameters of gelatin gel networks derived from transmission electron micrographs. The network gel replicas were obtained by a quick-freezing, deep-etching and rotary replication method adapted to the study of fragile physical networks by Favard P. et al. (Biol. Cell 67 (1989) 201-207). Stereo electron micrographs were taken by tilting the replicas at increasing angles between -35 to $+30^{\circ}$. A $3 \mathrm{D}$ reconstruction of the filamentous network was performed by stereoscopic and tomographic procedures. These procedures allowed us to measure the thicknesses of the replicas. The average length of filaments (triple helices) per unit volume and the average distance between filaments (mesh sizes of the networks) were derived for two polymer concentrations $(c=2 \%$ and $c=10 \%)$. The results are in full agreement with optical rotation and small-angle neutron scattering measurements.
\end{abstract}

\section{Introduction.}

One of the major difficulties in characterizing polymer gels is the description of their supramolecular structures. The gel structure has a primordial influence on the rheological properties and it is essentially controlled by the mechanisms of gelation. Difficulties in characterizing gel networks result from several causes : i) the very low content of polymeric material in the gel (a few percent), ii) the small size of the elements which usually build the network (between 10 and $1000 \mathrm{~nm}$ ), iii) the disordered character of the supramolecular assemblies. The most common methods for investigating gel networks are based on scattering techniques, using a combination of light, neutron and $X$-ray small-angle scattering, which have the advantages of being non-perturbing and of averaging over many structural elements.

(*) Author to whom correspondence should be addressed. 
However, interpretation of the scattering spectra in general is a delicate task. Thus, the direct observation of the three-dimensional network is one of the important steps for clearly identifying the structure. Only Transmission Electron Microscopy (TEM) may have a sufficient resolution to observe the microstructure of polymer networks. Considerable effort has been devoted to the development of reliable methods of preparation and observation of samples containing large amounts of solvent, particularly water. These methods are usually applied to biological systems, such as cells or tissues. The most reliable methods which are currently known are based on ultra-rapid freezing techniques [1] which have been shown to provide a better preservation of the specimens compared to chemical fixation. The rates of cooling which can be achieved with these methods are as high as $10^{4}$ to $10^{5} \mathrm{~K} / \mathrm{s}$, which are needed to freeze water in the vitreous state and thus to protect the specimen against damage caused by ice crystal formation.

Few observations of macromolecular gels using ultra-rapid freezing techniques are known, which we can briefly recall. The cryojet device uses liquid propane at $-180^{\circ} \mathrm{C}$ for quick freezing. This method was used by Zasadzinski et al. [2, 3] to study the morphology of (hydroxypropyl) guar gels. The same technique was applied to polyacrylamide gels by Hsu and Cohen [4] in order to derive the pore sizes of the gels. Another experiment of this type was carried out by Müller et al. [5] on sodium carboxymethyl cellulose aqueous gels which revealed a fine filamentous network of the system (thicknesses of $2-3 \mathrm{~nm}$ ). The so-called « mica sandwich technique » (the solution is placed between two freshly cleaved mica plates and plunged in liquid propane or nitrogen for quick freezing) was used by Hermansson et al. [6] for kappa-carrageenan gels in the presence of various salts. In these experiments, the fine details of the supramolecular assemblies, such as fine strands of double helices, and coarse aggregates of helices, could be visualized and studied in relation to changes in the rheological properties. The mica-sandwich technique was also chosen by Wade et al. [7] to observe a colloidal system (steroid/cyclohexane) in the physical gel state with a characteristic filamentous structure.

In a previous paper [8], we have reported a TEM observation of the structure of aqueous gelatin gels using a different freezing devices first proposed by Escaig [9], where the specimen was rapidly smashed against a copper block cooled with liquid helium to $4 \mathrm{~K}$ (the Cryoblock system). By applying a simple labelling technique to the surface of the gel prior to freezing, we have shown that we could clearly identify the surface which came first in contact with the copper plate and thus was frozen at the highest cooling rate. The network structure was preserved within a small distance from the surface ( circa $5 \mu \mathrm{m}$ ); ice crystals then appeared at larger depths and disrupted the structure.

In this paper, we present a quantitative analysis of the gelatin network based on TEM images obtained with the previous method. A three-dimensional reconstitution of the replicas was performed based on stereoscopic and tomographic procedures. With these procedures we have been able to measure the thickness of the replicas. Using a stereological analysis, we derived the average length of filaments per unit volume and the average distance between filaments, for two gel concentrations. The conclusions were confronted to optical rotation and neutron scattering measurements.

\section{Materials and methods.}

Gelatin Gel PREPARATION. - Gelatin was provided by Sanofi-Bio-Industries (Rousselot type). It is a lime processed demineralized ossein extract whose molecular characterization was published elsewhere [10]. The gelatin granules were swollen in distilled water for $2 \mathrm{~h}$ at $4{ }^{\circ} \mathrm{C}$ and then dissolved at $45^{\circ} \mathrm{C}$ during 30 min with a mild stirring. The $\mathrm{pH}$ of the solution initially close to the isoelectric point $(\mathrm{pH}=4.9)$ was adjusted to $\mathrm{pH}=8$ by adding a few drops of a 
$0.1 \mathrm{M} \mathrm{NaOH}$ solution in order to shield electrostatic attractive interactions between charged groups of the protein chains. The solutions was then cooled to room temperature $\left(20^{\circ} \mathrm{C}\right)$ and allowed to gel for $24 \mathrm{~h}$. Two concentrations were investigated : $2 \%$ and $10 \% \mathrm{~g} / \mathrm{g}$. In some specimens, latex spheres of diameter $0.09 \mu \mathrm{m}$ from Serva were added to the solution to serve as magnification markers.

REPLICA PREPARATION. - Small gelatin blocks circa $2.5 \times 2 \mathrm{~mm}$ and $3 \mathrm{~mm}$ of height were cut and attached with glycerol onto an $1.3 \mathrm{~cm}$ diameter copper disc which fitted the specimen holder of a Balzers $400 \mathrm{~T}$ apparatus. Crystals of $\mathrm{MgO}$ were deposited on the surfaces of the blocks to allow a surface recognition [8]. The preparation was rapidly transferred to a Cryoblock specimen holder (Reichert and Jung apparatus) and installed on the electromagnetic plunger. Quick-freezing was achieved by smashing the sample surface against a high purity copper block cooled with liquid Helium to $4 \mathrm{~K}$ and maintained in vacuum. The frozen samples were first stored in liquid nitrogen and then transferred to the Balzers $400 \mathrm{~T}$ vacuum evaporator $\left(2 \times 10^{-5} \mathrm{~Pa}\right)$. The evaporator was equipped with a cryomicrotome device cooled to liquid nitrogen temperature. The scraping was performed very slowly while the gel was maintained at $-100^{\circ} \mathrm{C}$, in order to avoid or reduce any fracturing phenomena and to obtain even surfaces. The gel was then etched $\left(10 \mathrm{~min}\right.$ at $\left.-90^{\circ} \mathrm{C}\right)$. Rotary replication for platinum evaporation took place at an angle of $45^{\circ}$ and at $90^{\circ}$ for carbon evaporation. The replicas were then cleaned and picked on copper electron microscope grids whose mesh size (400 copper bars per inch) was chosen because of the fragility of the replicas.

RePlica examination. - Replicas were examined with a Philips 300 electron microscope equipped with a goniometric stage. Electron micrographs were taken by tilting the replicas at increasing angles from -35 to $+30^{\circ}$ five by five degrees for the three-dimensional reconstitution.

THREE-DIMENSIONAL RECONSTITUTION. - The two main methods used for the three-dimensional reconstitution are the stereoscopic method [11] and the tomographic method. The principles of these methods are briefly described.

The first method used in electron microscopy was the stereoscopic method $[12,13]$. It requires the acquisition of two tilted views of the specimen. A qualitative reconstitution can then be obtained by observing the stereo-pair with a stereoscope. Quantitative stereoscopy can be performed with the help of computers. A preliminary microcomputer solftware was developed by Bonnet et al. [14] which allows the height measurements to be made from the coordinates of homologous details, these coordinates being obtained with a digitalizer. This software has been extended for digital stereoscopy to work on an image analyzer (BIO 500 , BIOCOM, Les Ulis, France). This new software allows the geometric registration of image pairs, the $3 \mathrm{D}$ visualization of the stereo-pairs through the anaglyph procedure (with red/green spectacles) and the height measurements of specific details defined on the screen of the analyzer [15]. This procedure was used in this study for analyzing the $c=2 \%$ gel replica with tilt angles at $\pm 10^{\circ}$.

The tomographic procedure is an extension of the stereoscopic method which has to be used for specimens with complicated structures. A large number of projections (images) are recorded at different viewing angles with the goniometer stage operating in the single axis mode. The 3D reconstitution of the specimen is performed by computer, according to algorithms similar to those of X-rays medical tomography. Ideally, images should be recorded through the whole solid angle (tilt angles between $-90^{\circ}$ and $+90^{\circ}$ ). However, the mechanical limitations of the goniometer stage and the mesh size of the grids have reduced the tilt angles for our replicas to $\pm 35^{\circ}$. About 15 images have been recorded with tilt angles increments of $5^{\circ}$ 
for the gel concentration $c=10 \%$. Micrographs were digitalized into $512 \times 512$ pixels, 256 grey levels. After image registration, $128 \times 128$ pixels areas were submitted to tomographic reconstitution. The software for this reconstitution [16] is a combination of different algorithms already described in the literature [17-20] which have been slightly modified : the Extended Field Filtered Simultaneous Iterating Reconstruction Technique (EFFSIRT) and the Extended Field Algebraic Reconstruction Technique (EFART). All the algorithms rely on the general principle of back projection : the imaging process consists in a projection of the object to the images ; the reconstitution consists in the reverse process : images are back projected to the volume and different projections are added together, with some mathematical tricks, in order to take into account the geometry of the acquisition set-up. Pseudo-images are then computed from this preliminary reconstructed object which can then be refined according to the difference between computed and experimental projections.

For this study the two algorithms were applied and they gave similar results within the accuracy of this analysis. The 3D reconstructed objects were defined as $128 \times 128 \times 128$ volume elements (voxels). The $3 D$ visualization was performed on a SUN 4/370 workstation, either with the help of routines contained in the SUNVISION software or with home made routines. The different visualization modes are : slicing in any direction, $(x, y),(x, z)$, $(y, z)$ or oblique ; surface visualization through ray tracing and object rotation and movies. Measurements of the specimen thickness were performed on the reconstituted 3D objects.

\section{Results.}

$c=2 \% \mathrm{GEL}$. - In figure 1 we show a stereo pair with tilt angles $\pm 6^{\circ}$ of the gel. The views were taken in the superficial area of the specimen (within $5 \mu \mathrm{m}$ from the surface) which was previously shown to correspond to the best freezing conditions [8]. Small crystals of $\mathrm{MgO}$ can be seen on the image : this proves that the observed area is located near the surface. Some latex spheres also appear on the micrographs.

The replica shows a dense filamentous network with tightly entangled strands. The apparent thickness of the strands is due to the shadowing. This can be better observed in figure 2 , where a processed image of the network is shown : the smallest details were reinforced according to a local contrast enhancement procedure. The denser inner core is the Pt coated basic filament of the gel network. Its diameter is about $1-2 \mathrm{~nm}$. The outer carbon coating appears more transparent to the electron beam. The thickness of the outer layer is of the order of $10-20 \mathrm{~nm}$. Some filaments are so close to each other that they seem to form in places bundles with apparent larger diameters. However, there is no clear tendency to observe a parallel alignment of the filaments over large distances. The filaments keep a straight orientation over distances which do not exceed $100 \mathrm{~nm}$. From the stereo-view of figure 1, using the stereoscopic analysis, we were able to measure the height of the replica, by selecting homologous points located at the lower and the upper parts. We found an average value of $h=0.10 \pm 0.01 \mu \mathrm{m}$. This value was used (see below) for the determination of the characteristic parameters of the network derived from the images.

Figure 3 shows a series of tilted images of an enlarged detail of the network, between $-35^{\circ}$ and $+30^{\circ}$. One can follow the relative positions of the filaments as the sample is tilted. The tilting effect would be more pronounced if larger inclination angles could be achieved.

$c=10 \%$ GEL. - Figure 4 shows the replica of the gel. The magnification in figure $4 \mathrm{a}$ is identical to the previous micrograph. The network appears denser and more homogeneous than the previous one. The thickness of the replica could not be determined by the stereoscopic method because the structure is too complicated and the definition of homologous details in the stereo-pair was not reliable. Therefore, we used the tomographic method to measure the 

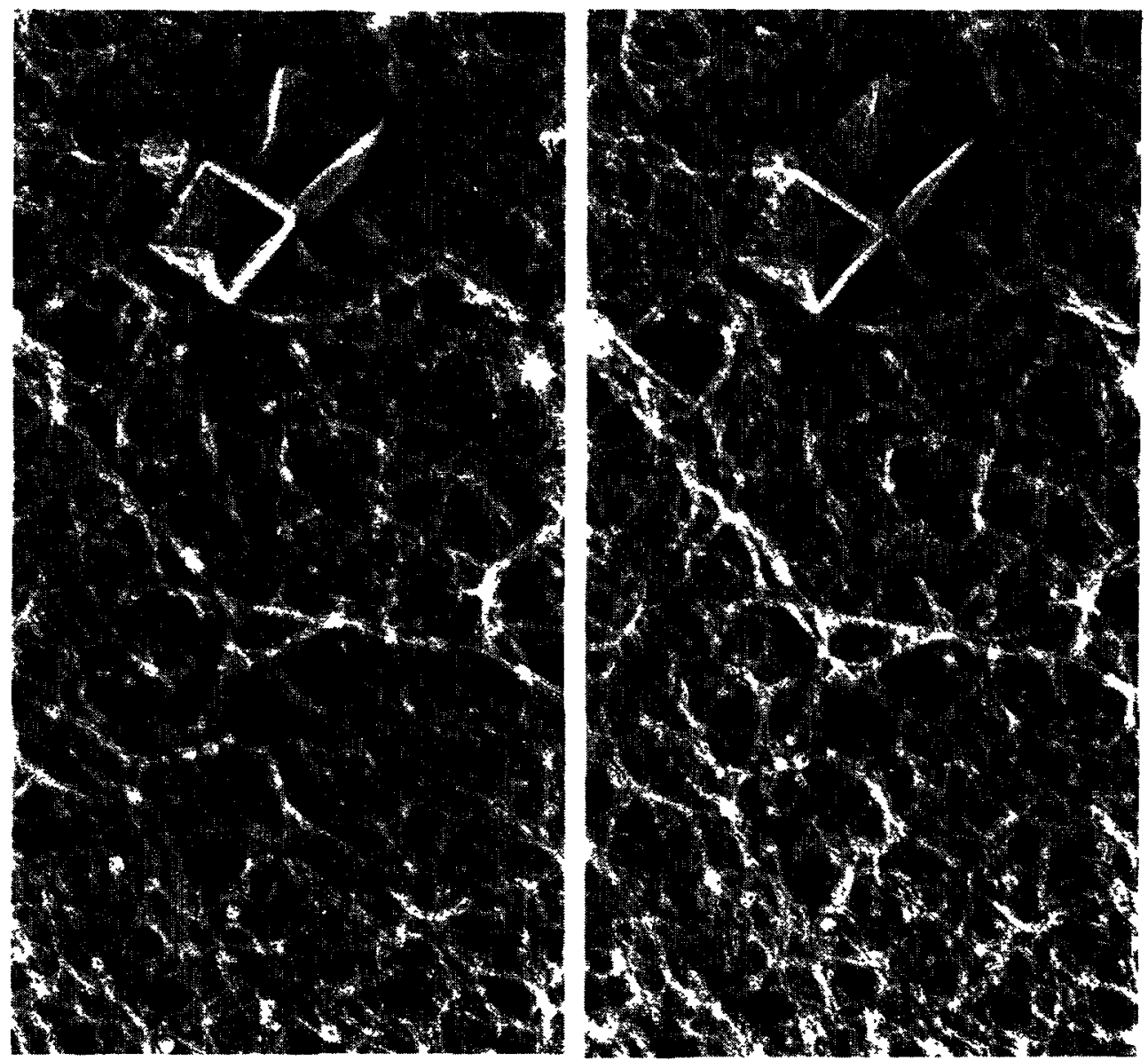

Fig. 1. - Stereo-pair of a quick-frozen and deep-etched replica of a gelatin gel $c=2 \%$. The 3D organization of the network is observed. The network is dense and filamentous. MgO crystals and latex spheres are seen in the images and serve as markers. Looking at the image pair with a stereoscope allows to perceive the $3 \mathrm{D}$ effect. Micrographs are printed as negative images. Magnification $: \times 77000$.

thickness of the replica. A series of tilted images was taken which is shown in figure 5. An overview of the reconstructed volume is displayed in figure 6 . A series of vertical sections $(x, z)$ normal to the replica surface, cut through the reconstructed $3 \mathrm{D}$ object, are displayed in figure 7 . Due to the limits of the solid angle within which the projections were obtained, the quality (resolution) of the reconstruction is not sufficient for analyzing the network in detail, but it is acceptable for deriving the needed information on the thickness of the replica, which was found to be $h=0.015 \pm 0.005 \mu \mathrm{m}$.

THE NETWORK PARAMETERS. - We continued the analysis on the $2 \mathrm{D}$ projections of the replica, which are the images taken in the horizontal position of the microscope grids. For this analysis, it was essential to know the thickness of the replicas which could be derived from the previous procedures. The $2 \mathrm{D}$ analysis is based on well known principles in stereology [21]. We consider the network as a system of lines (filaments) in volume, as schematically shown in figure 8. The system of lines may consist of straight, curved, continuous or broken lines, oriented and located randomly throughout the volume. Let $L_{\mathrm{y}}$ be the total length of random 


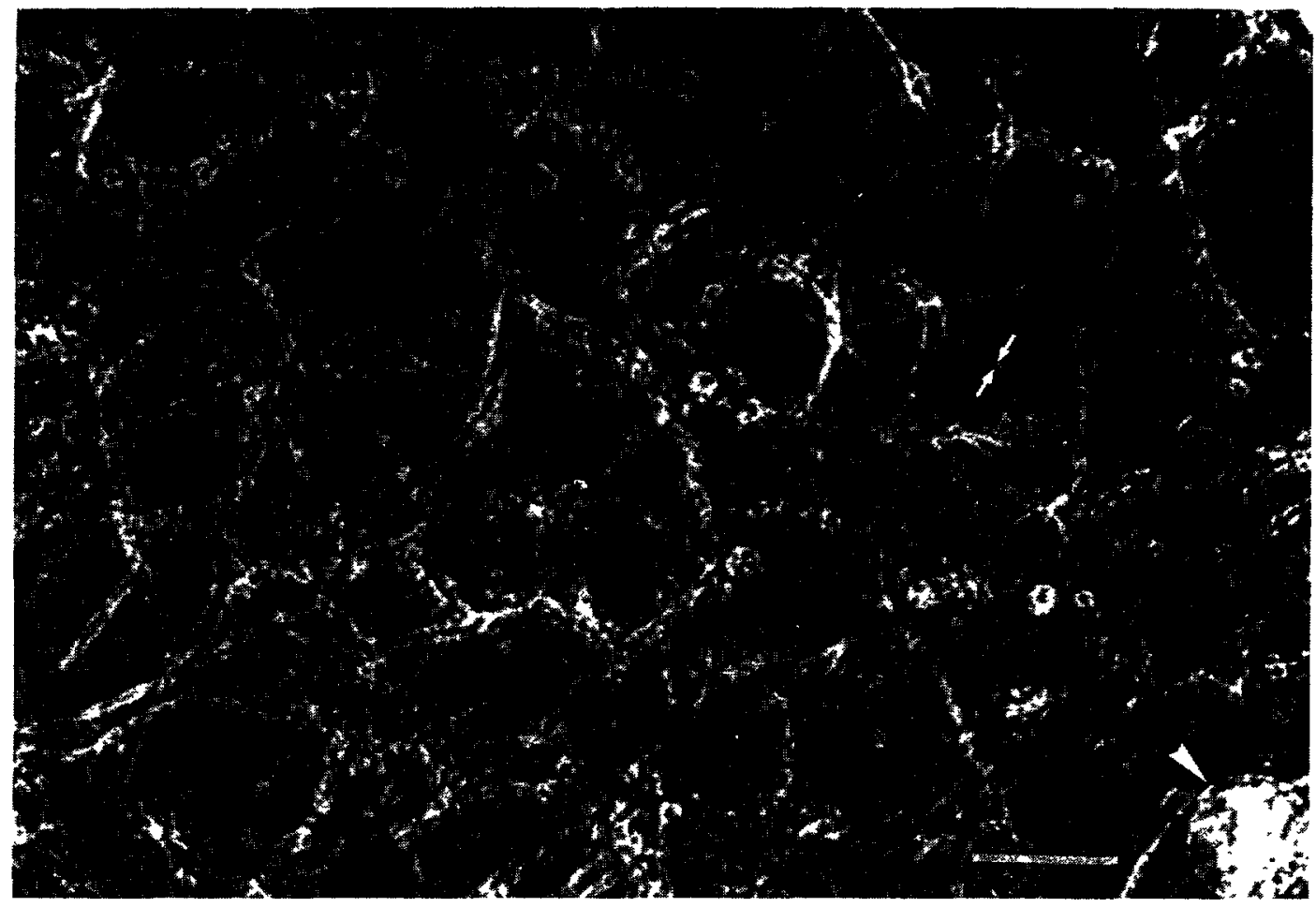

Fig. 2. - Digitally processed enlarged part of the replica of the $c=2 \%$ specimen. The local contrast enhancement procedure was used to reveal the fine details of the original picture. The arrow shows a latex sphere. The thin inner core of a filament is visible between the thin arrows. Bar: $0.1 \mu \mathrm{m}$.

lines per unit volume. As shown in figure 8 , we consider the intersection points of these lines with a test plane.

Let $N_{\mathrm{a}}$ be the number of points of intersection per unit area with a test plane. If one assumes that all angles of orientation between lines and test planes are equally possible, then one establishes the relation :

$$
L_{\mathrm{v}}=2 N_{\mathrm{a}}\left(\mu \mathrm{m} / \mu \mathrm{m}^{3}\right) \text {. }
$$

The TEM image is the projection of a volume element of thickness $h$. Thus, the points of intersection of the test planes with the filaments can be counted on the TEM image as the intersections of the projected filaments with a test line. Let $N_{1}$ be the number of intersection points per unit length of the test line in the projected image. Then :

$$
N_{1}=N_{\mathrm{a}} h\left(\mu \mathrm{m}^{-1}\right)
$$

and

$$
L_{\mathrm{v}}=2 \frac{N_{1}}{h}\left(\mu \mathrm{m} / \mu \mathrm{m}^{3}\right)
$$

Another length can be deduced from the TEM images which is the nearest neighbors distance 


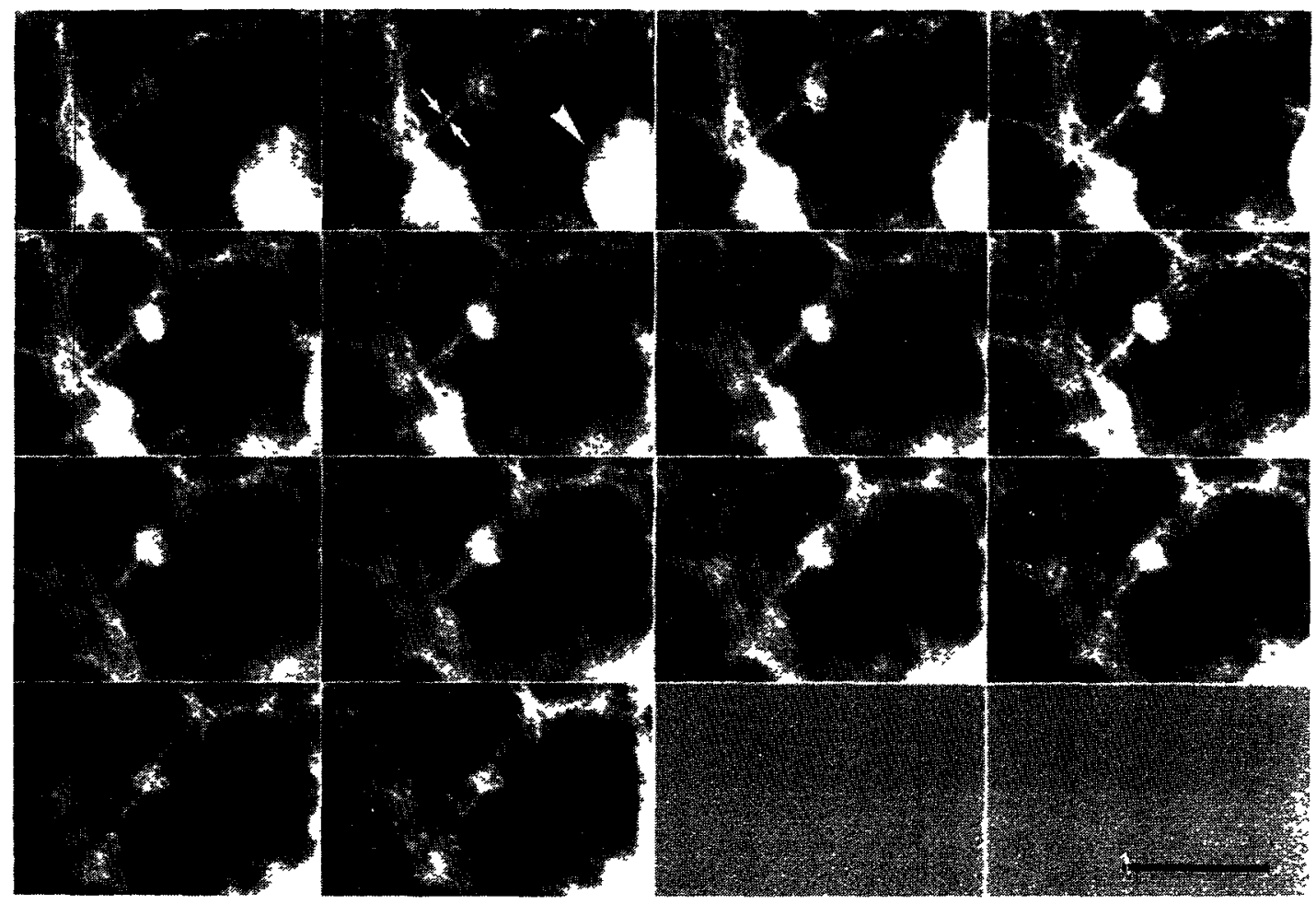

Fig. 3. - Tilted series of the replica of the $c=2 \%$ specimen. Fourteen images corresponding to the same region of the specimen are displayed with tilt angles of the holder from $-35^{\circ}$ (upper left image) to $+30^{\circ}$ (lower right image). Through the parallax effect, the three-dimensional information is coded in the image series and the computer reconstruction allows to restore it in a qualitative way. By looking at the picture gallery one has the perception of depth or thickness of the replica. The arrow head shows a latex sphere. The thin arrows indicate the inner core of a filament. Bar: $0.1 \mu \mathrm{m}$.

$\Delta$ between the points of intersection of filaments with the test planes. One can show that :

$$
\Delta=\left(\frac{h}{4 N_{1}}\right)^{1 / 2}=\left(2 L_{v}\right)^{-1 / 2}(\mu \mathrm{m})
$$

Considering the micrographs of the $c=2 \%$ and $c=10 \%$ gels, we counted the number of intersections of randomly oriented test lines with the projected filaments of the network. The great density of the network and the loss of resolution caused by the coating obviously limit the accuracy of this analysis. Nevertheless, we were able to derive the following values :

- for $c=2 \%, \quad N_{1}=70 \pm 5 \mu \mathrm{m}^{-1}$

Taking $h=0.10 \pm 0.01 \mu \mathrm{m}$, one has :

$$
L_{\mathrm{v}}^{\mathrm{TEM}}=1400 \pm 250 \mu \mathrm{m} / \mu \mathrm{m}^{3} \quad \text { and } \quad \Delta^{\mathrm{TEM}}=0.02 \pm 0.01 \mu \mathrm{m}
$$



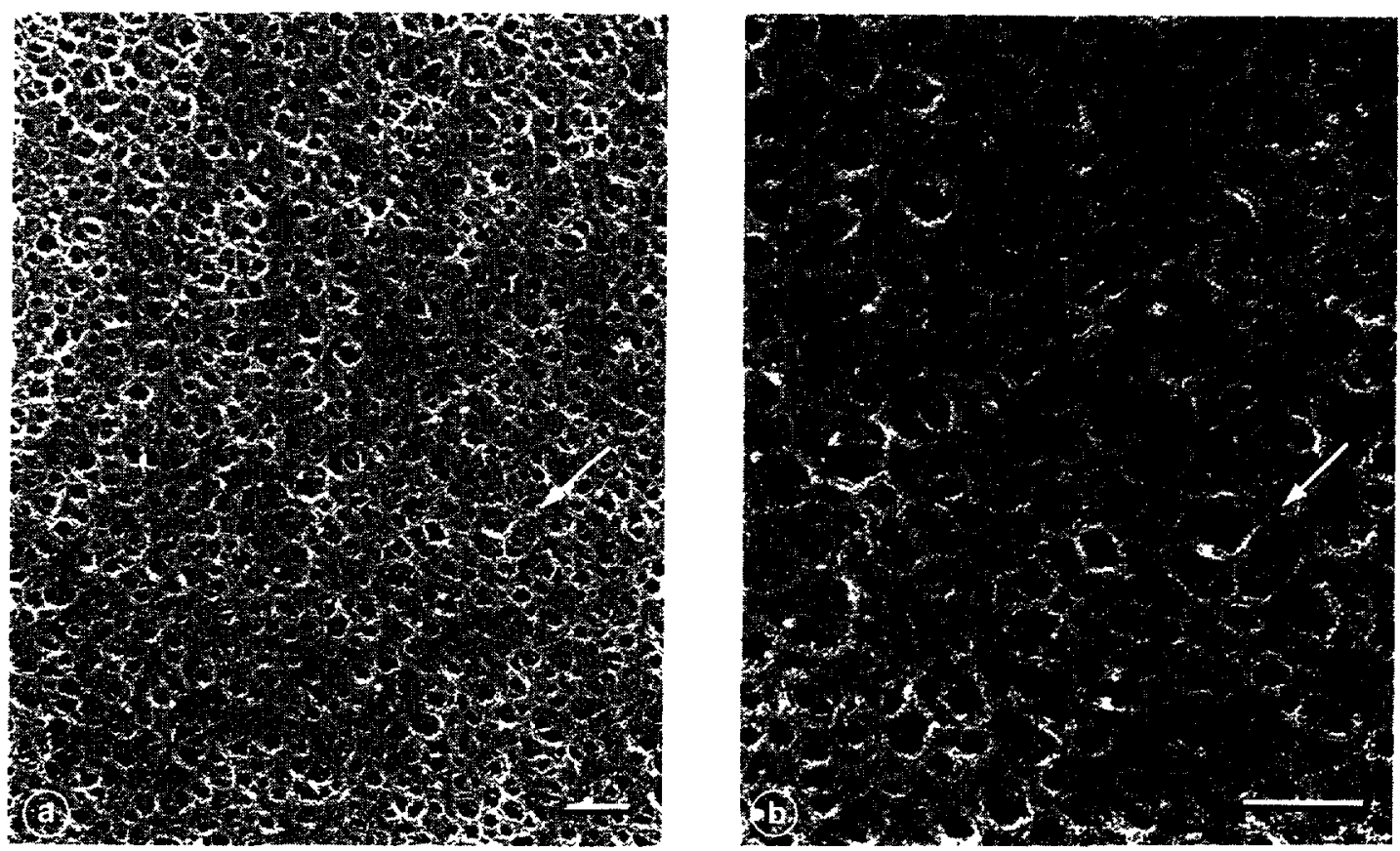

Fig. 4. - Replicas of the $c=10 \%$ gelatin gel. The dense filamentous network is more homogeneous than the $c=2 \%$ gel. Negative images. a) magnification $\times 77000$ (as in Fig. 1). b) a higher magnification $\times 154000$. In the region shown by the arrows the depth of the replica appears distinctly.

- for $c=10 \%, \quad N_{1}=80 \pm 5 \mu \mathrm{m}^{-1}$

Taking $h=0.015 \pm 0.005 \mu \mathrm{m}$, then :

$$
L_{\mathrm{v}}^{\mathrm{TEM}}=10500 \pm 300 \mu \mathrm{m} / \mu \mathrm{m}^{3} \quad \text { and } \quad \Delta^{\mathrm{TEM}}=0.007 \pm 0.005 \mu \mathrm{m} .
$$

These values can be compared with the data derived from spectroscopic methods.

\section{Discussion.}

Gelatin gels are formed by lowering the temperature of semi-dilute solutions $(c>1 \%)$ initially at $45^{\circ} \mathrm{C}$, in the sol state, below $30^{\circ} \mathrm{C}$ where the coils undertake a conformational coil to helix transition. The helices are of the collagen type, they are triple left-handed helices wrapped in a super right-handed helix, stabilized by hydrogen bonds. Helices are nucleated along the individual chains in the proline rich sequences. When helices are nucleated on segments belonging to three different strands, either from two or three different chains closely entangled [22], they grow and form a junction or a filament of the network.

The average length of triple helices $L_{v}$ can be estimated from optical rotation measurements (OR). Indeed, the helix amount $X$ (or the fraction of residues which have been converted from random coil to helical conformation in the gel) is known for different temperatures and gelatin concentrations. It has been shown [23] that the helix amount $X$ increases continuously with time and depends on the thermal history of the samples. An approximate value for the helix amount after 24 hours at room temperature is $X=40 \%$. The collagen type triple helix has a mass per unit length of $1000 \mathrm{~g} / \mathrm{nm} / \mathrm{mole}$. Thus, one can derive the average triple helix length 


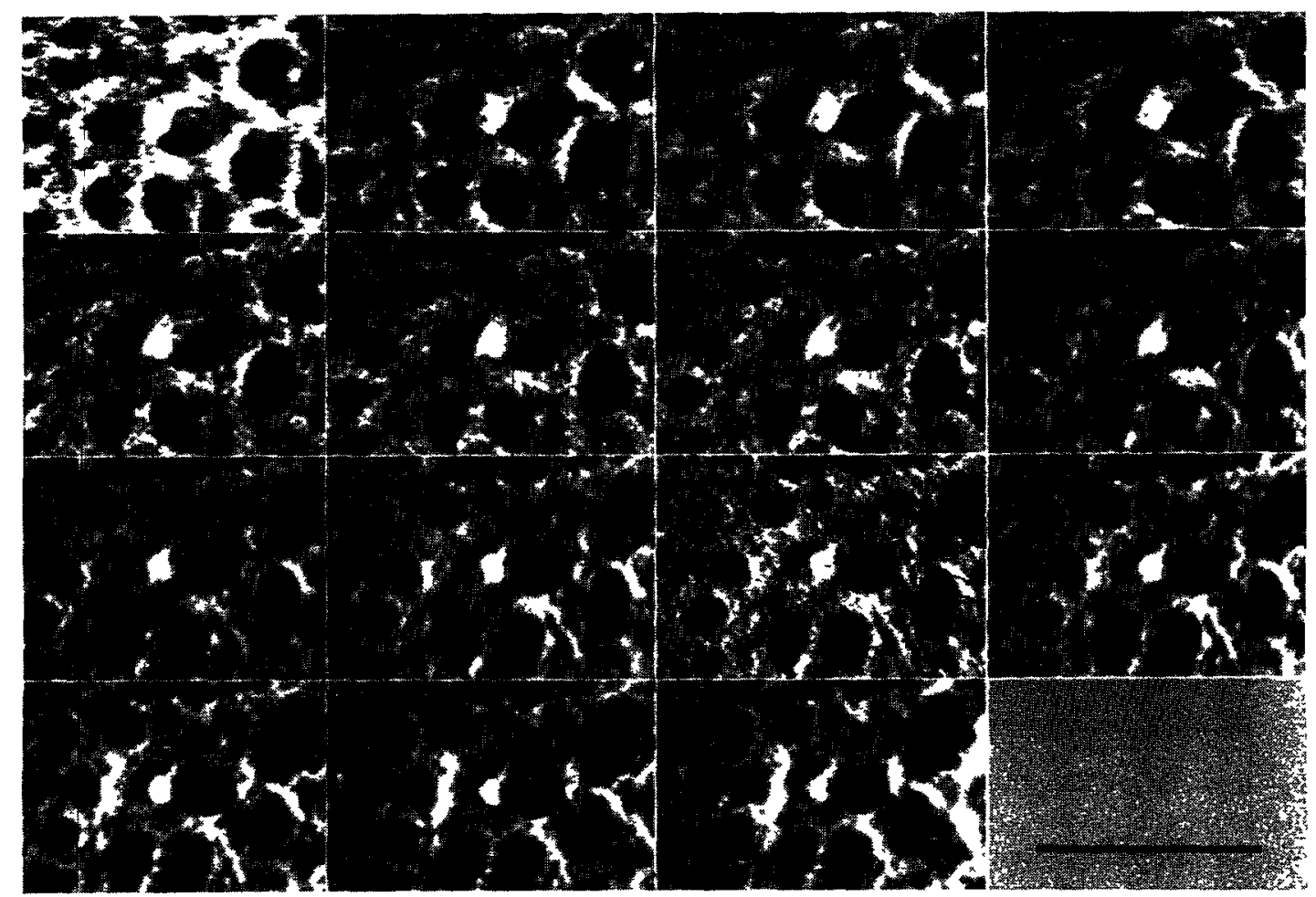

Fig. 5. - Tilted images of the $c=10 \%$ specimen. The tilt angles range from $-35^{\circ}$ (upper left image) to $+35^{\circ}$ (lower right image) with a step of $5^{\circ}$. Looking carefully, one can follow from one image to the next, the progressive displacement of a detail of the image due to the tilting effect. The full width of each image is $0.145 \mu \mathrm{m}$. Bar : $0.1 \mu \mathrm{m}$.

per unit volume :

$\begin{array}{lll}\text { for } & c=2 \%, & L_{\mathrm{v}}^{\mathrm{OR}}=4800 \mu \mathrm{m} / \mu \mathrm{m}^{3} \\ \text { for } & c=10 \%, & L_{\mathrm{v}}^{\mathrm{OR}}=24000 \mu \mathrm{m} / \mu \mathrm{m}^{3}\end{array}$

The order of magnitude for $L_{\mathrm{v}}^{\text {TEM }}$ derived from the replicas is in agreement with the calculation of $L_{\mathrm{v}}^{\mathrm{OR}}$ The difference between the two values may be interpreted in several ways :

i) the network is dense. The Pt-C coating enhances the contrast to electron scattering, but obscures the fine details, so that several helices close to each other may be counted within one filament, which then underestimates the value of $N_{1}$;

ii) the thickness of the replicas $h$ is comparable to the average distance between filaments $\Delta(h \approx 5 \Delta$ for $c=2 \%$ and $h \approx 2 \Delta$ for $c=10 \%)$. Minor surface defects as compared to bulk properties of the network may have some influence ;

iii) errors are introduced in measuring the thickness of the replicas by the processing of the images. If a greater number of tilted images were recorded, a more precise geometric registration of the different images could be achieved.

The average distance between filaments is another parameter which we determined from this analysis. One first may note that on the TEM images the filaments have a large distribution of lengths, especially in the more dilute gel. It is not possible to derive quantitative values for the 


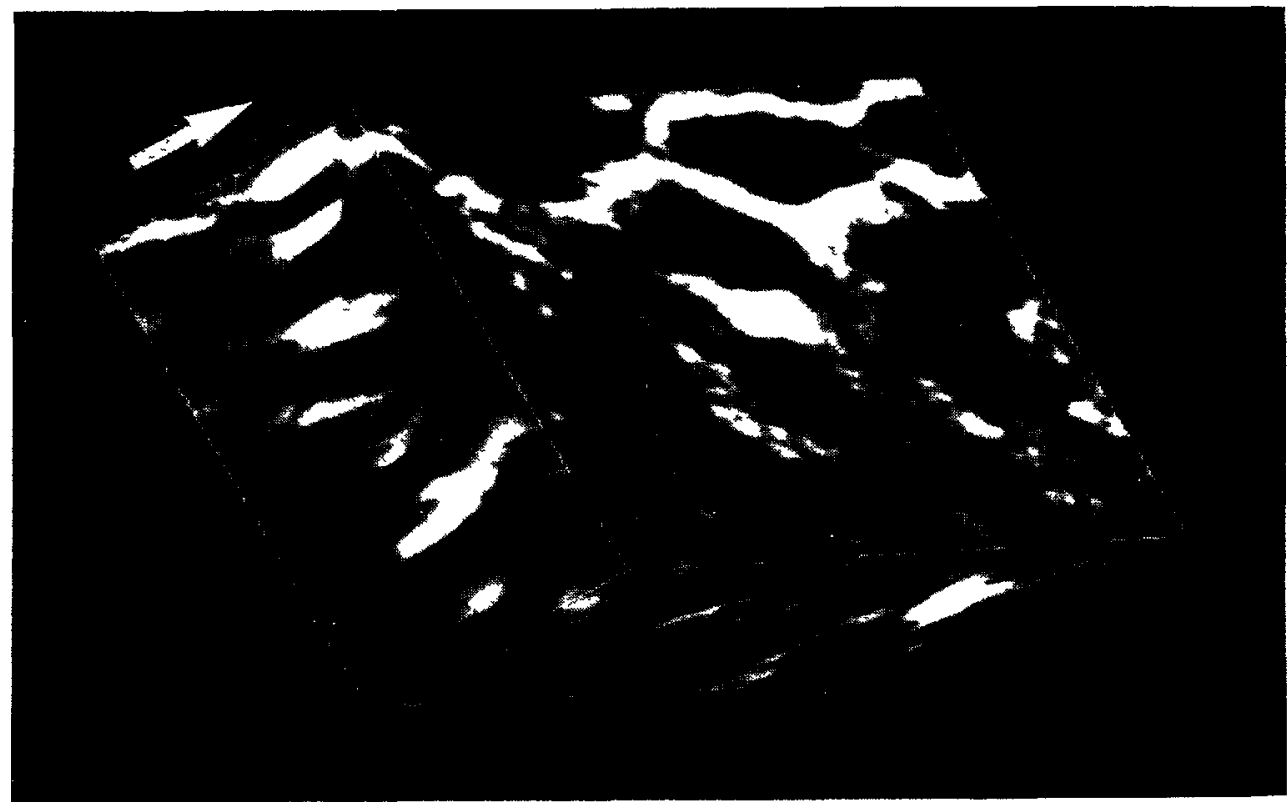

Fig. 6. - Overview of the reconstructed volume (cube of $0.145 \mu \mathrm{m}$ side) of the $10 \%$ gel from the $2 \mathrm{D}$ images seen in figure 5. Once the 3D object is built in the computer memory several procedures can be used for displaying it onto the screen, where the object can be rotated in any direction and observed through several modes : surface, transparent... The arrow shows the direction of the electron beam when the specimen is observed in the untilted position.

distribution, but it appears that the network meshes may range between $10 \mathrm{~nm}$ and $100 \mathrm{~nm}$, for instance for $c=2 \%$. The average mesh size of the network can be compared with data provided by the small angle neutron scattering (SANS) spectra of the gels. The scattering spectra in the sol and gel states are shown in figure 9 for $c=5 \%$, where light and neutron scattered intensities were plotted versus the scattering vector $q$. In these experiments, the gels have been matured for a longer period ( 7 days) during which slightly more helices $(45 \%)$ have been created. However, the striking feature of the figure is that the sol and the gel states have almost the same scattering spectra. The (minor) differences have been interpreted in the following way [24]:

i) a small increase in the light scattering data (for scattering vectors $q<0.03 \mathrm{~nm}^{-1}$ ) shows that the gel has a larger amount of inhomogeneities as compared to the sol state, although they remain clear and transparent. Such inhomogeneities also appear on the TEM replicas;

ii) in the intermediate scattering vector range, $0.05<q<0.5 \mathrm{~nm}^{-1}$, one can derive the correlation length of the concentration fluctuations using the theoretical models based on the scaling laws [25], that we may call the equivalent «mesh size » of the transient network of entangled chains. This treatment is valid for semi-dilute solutions of random coils. The scattering spectra $I(q)$ has a Lorentzian shape with a characteristic length $\xi$. In the sol state $\left(T=45^{\circ} \mathrm{C}\right.$ ) for the same concentrations as in the present investigation :

$$
c=2 \%, \xi=5 \mathrm{~nm} \text { and for } c=10 \%, \quad \xi=2 \mathrm{~nm} .
$$

As the spectra are quasi identical in this range of scattering vectors, this analysis applied to the 


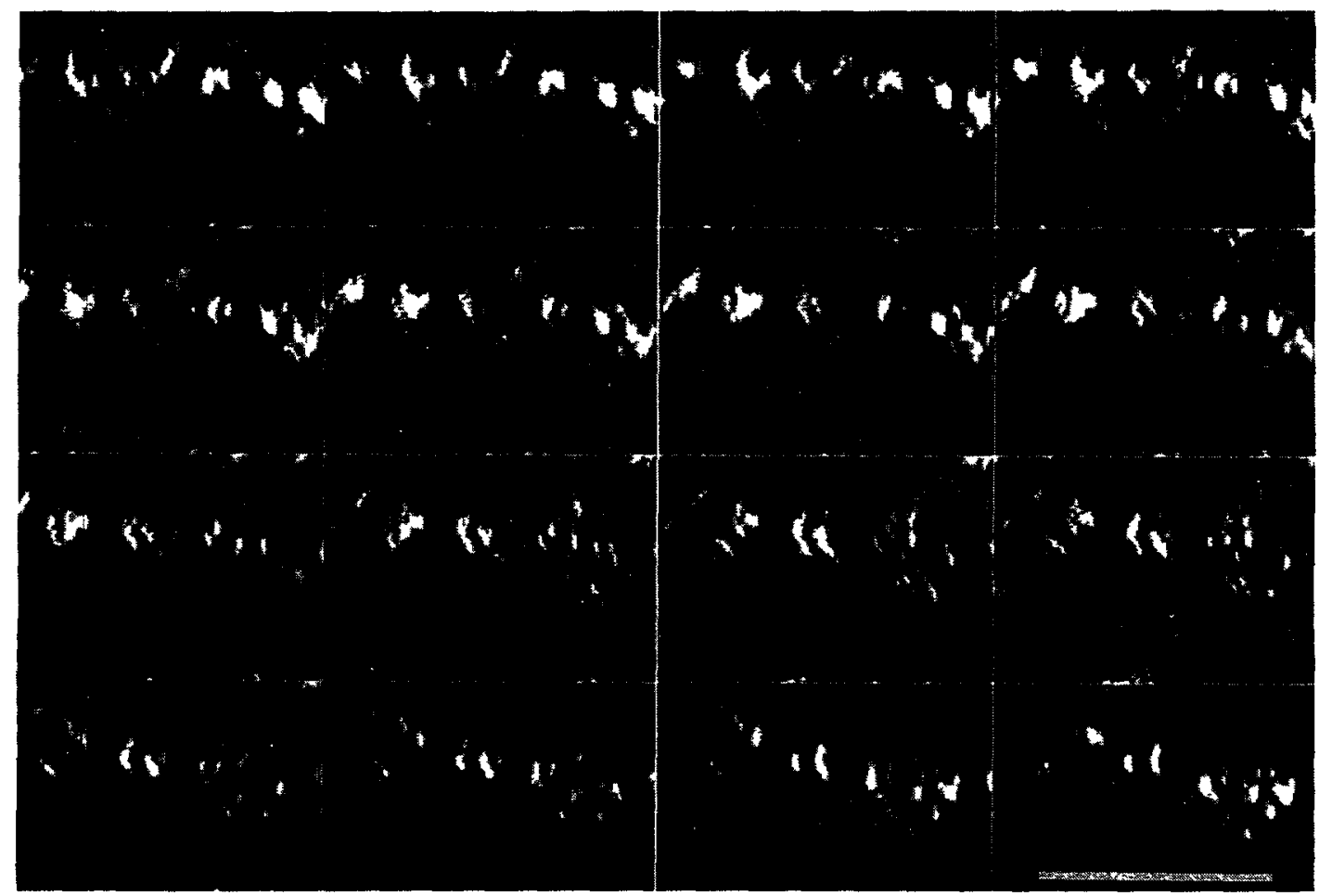

Fig. 7. - Another mode of visualization of the reconstructed object seen in figure 6. These images are « vertical » slices of the object, i.e. normal to the surface of the plane of the sample. These views allow to measure the local thickness of the replica which was the needed information. Note that the real material is in white, dark pixels correspond to the holes of the network. Grey pixels correspond to artefacts produced by the limitations of the reconstruction procedure (slight misregistrations of the images, limited number of projections...). Bar : $0.1 \mu \mathrm{m}$.

gel state gives nearly the same values for the correlation lengths of the gel network (in fact the $\xi$ values are about $10 \%$ higher in the gel compared to the sol).

Comparing the values of $\xi$ and $\Delta$, we find $\Delta>\xi$. One can speculate on the origin of this inequality. First of all, the two parameters $\Delta$ and $\xi$ do not rigorously represent the same quantities : in the TEM images, only the network of triple helices is apparent. As stated in the beginning, the coils are beyond the resolution of the electron microscope. In the gel state however, large fractions of the chains are in random coils, so that the gel structure contains portions of triple helices $(40 \%)$ and random coils $(60 \%)$, which are tightly interpenetrated. By SANS one cannot separate the contribution of the random coils from the triple helices. The correlation lengths determined by SANS are distances between nearest neighbors strands, either coils or helices. Thus, one normally should expect the « mesh sizes » $\xi$ measured by scattering techniques to be smaller than the average distances $\Delta$ measured by TEM (network of triple helices). The second reason for this difference is simply related to the determination of the length $L_{\mathrm{v}}^{\text {TEM }}$ deduced from the replicas. If we calculate $L_{\mathrm{v}}^{\mathrm{OR}}$, then derive $\Delta^{\mathrm{OR}}$ we find from equation (4) : $\Delta^{\mathrm{OR}} \approx 10 \mathrm{~nm}$ (instead of $20 \mathrm{~nm}$ ) and $\Delta^{\mathrm{OR}} \approx 4 \mathrm{~nm}$ (instead of $7 \mathrm{~nm}$ ), for the two concentrations. These values are closer to the $\xi$ values and are as expected higher.

iii) The slope of the scattering spectra, for the higher scattering vectors, $0.3 \leqslant q<3 \mathrm{~nm}^{-1}$, allows to determine the average radius of cross-section $R_{c}$ of the strands in the sol and gel 

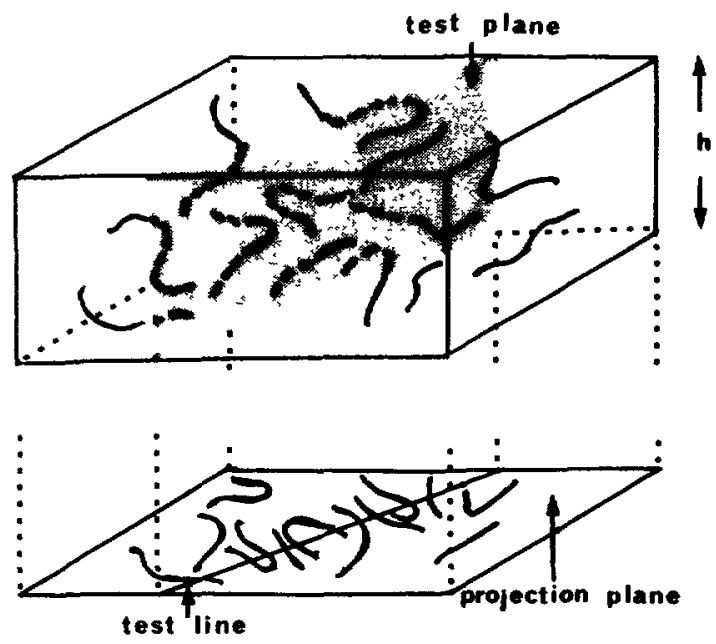

Fig. 8. - Schematic drawing of the microstructural features in a foil of thickness $h$ and their projection onto a plane. The system consists of random lines. A vertical slice - a test plane - cuts the lines through the foil, the number of intersections per unit area of the test plane being $N_{\mathrm{a}}$. In the projected image, one counts the number of intersections per unit length of the test line, $N_{1}$. Under certain assumptions, it is possible to deduce the magnitude of quantities in the foil from measurements on projected images.

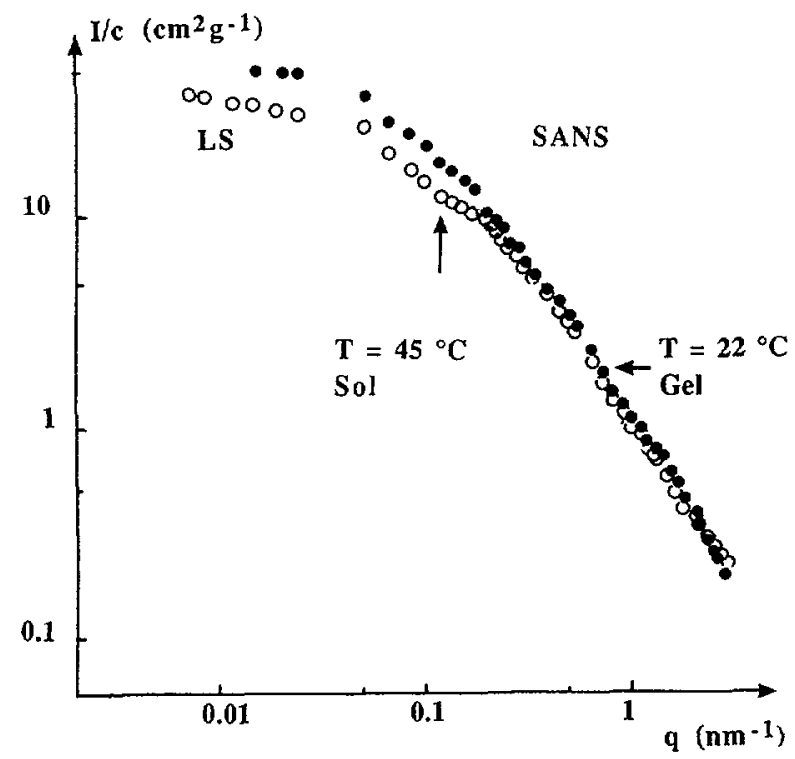

Fig. 9. - Light scattering (LS) and small-angle neutron scattering (SANS) of gelatin solutions in the sol $\left(T=45^{\circ} \mathrm{C}\right)$ and in the gel states $\left(T=22^{\circ} \mathrm{C}\right.$, after 7 days). The scattering spectra are almost identical except towards the smaller scattering vector range. The correlation lengths and the cross-section of individual coils or filaments are deduced from the spectra (from Ref. [24]). 
states. We found respectively, $R_{\mathrm{c}}^{\mathrm{sol}}=0.28 \mathrm{~nm}$ and $R_{\mathrm{c}}^{\mathrm{gel}}=0.43 \mathrm{~nm}$. The cross-section of the coils is in agreement with the molecular composition of the protein chains and the cross-section of the strands in the gel state is an intermediate value between single coils and triple helices (for the collagen type triple helix $R_{\mathrm{c}}=0.6 \mathrm{~nm}$ ). Thus, the scattering spectrum clearly indicates that crystalline type, large bundles of triple helices are not present in the gel, thus corroborating the overall analysis proposed for the TEM images.

\section{Conclusion.}

This paper reports a quantitative analysis of the 3D network of a physical aqueous gel, the gelatin gel. This analysis is based on stereoscopic and tomographic methods applied to a 3D reconstitution of the gel network. The results, compared to independently derived structural parameters of the gel, clearly demonstrate that the methods of replica preparation by quickfreezing, deep-etching and rotary shadowing, of stereo TEM observation and of data processing were fully valid, within the limits discussed above. The visual observation of the network provides incomparable support to interpretations of the spectroscopical measurements. Reliable methods for image processing and reconstruction of complex three-dimensional objects are rapidly developing.

\section{References}

[1] Heuser J. E., Reese T. S., Dennis M. J., Yan Y., Evans L., J. Cell Biol. 81 (1979) 275 ; Heuser J. E., Methods in Cell Biology, J. N. Turner Ed., 22 (Acad. Press, 1981) p. 97.

[2] Zasadzinsky J. A. N., Chu A., Prud'homme R. K., Macromol. 19 (1986) 2960.

[3] Zasadzinsky J. A. N., Kramer J., ChU A., Prud'homme R. K., Chem. Eng. Commun. 52 (1987) 283.

[4] HsU T.-P., COHEN C., Polymer 25 (1984) 1419.

[5] Müller T., Hakert H., ECKert Th., Colloid Polym. Sci. 267 (1989) 230.

[6] Hermansson A.-M., Eriksson E., Jordansson E., Carbohydrate Polym. 16 (1991) 297.

[7] Wade R. H., Terech P., Hewat E. A., Ramasseul R., Volino F., J. Colloid Interface Sci. 114 (1986) 442.

[8] Favard P., Lechaire J.-P., Maillard M., Favard N., Djabourov M., Leblond J., Biol. Cell 67 (1989) 201.

[9] Escaig J., J. Microsc. 126 (1982) 221.

[10] Djabourov M., Papon P., Polymer 24 (1983) 539.

[11] TuRner J. N., Methods in Cell Biology, 22 (Academic Press, London, 1981) p. 1.

[12] See for instance: FAVARD N., FAVARD P., Analysis of Organic and Biological Surfaces (John Wiley and Sons, NY, 1984) p. 351.

[13] See for instance : PORTER K. R., STERN M. E., reference [11], p. 53.

[14] Bonnet N., Quintana C., Favard P., Favard N., Biol. Cell. 55 (1985) 125.

[15] Beorchia A., Ploton D., Menager M., Lebonvallet S., Thiry M., Bonnet N., J. Microsc. 163 (1991) 221.

[16] Kaplan H., Bonnet N., Beorchia A., Heliot L., El Hila H., Ploton D., Menager M., Proc. Coll. Franco-Iberique Electron Microsc. Barcelona (1991) 295.

[17] Gordon R., Bender R., Herman G., J. Theor. Biol. 29 (1970) 471.

[18] Gilbert P., Proceed. R. Soc. London B 182 (1972) 89 and J. Theor. Biol. 36 (1972) 905.

[19] Crowther R., Klug A., Nature 251 (1974) 490.

[20] Herman G., Image reconstruction from projections (Academic Press, N.Y., 1980).

[21] Underwood E. E., Quantitative stereology (Addison-Wesley Publish. Company, Reading, Massachusetts, 1970). 
[22] Ross-Murphy S. B., Polymer 33 (1992) 2623.

[23] Djabourov M., Leblond J., Papon P., J. Phys. France 49 (1988) 319.

[24] Pezron I., Herning T., Djabourov M. and Leblond J., Physical Networks, Ross-Murphy S. B. and Burchard W. Eds. (Elsevier Applied Science, London, 1990) Ch. 18.

[25] Pezron I., Djabourov M., Leblond J., Polymer 17 (1991) 3201. 